\title{
A Scalable Continuous Vortex Reactor for Gram to Kilo Scale for UV and Visible Photochemistry
}

Darren S. Lee ${ }^{a}$, Medhat Sharabi ${ }^{b}$, Richard Jefferson-Loveday ${ }^{b}$, Stephen J. Pickering ${ }^{b}$, Martyn Poliakoff*a, Michael W. George*a,c

${ }^{a}$ School of Chemistry, University of Nottingham, University Park, NG7 2RD, UK

${ }^{b}$ Department of Mechanical and Manufacturing Engineering, University of Nottingham, University Park, NG7 2RD, UK

'Department of Chemical and Environmental Engineering, The University of Nottingham Ningbo China, 199 Taikang East Road, Ningbo 315100, China

mike.george@nottingham.ac.uk; martyn.poliakoff@nottingham.ac.uk 
TOC Image

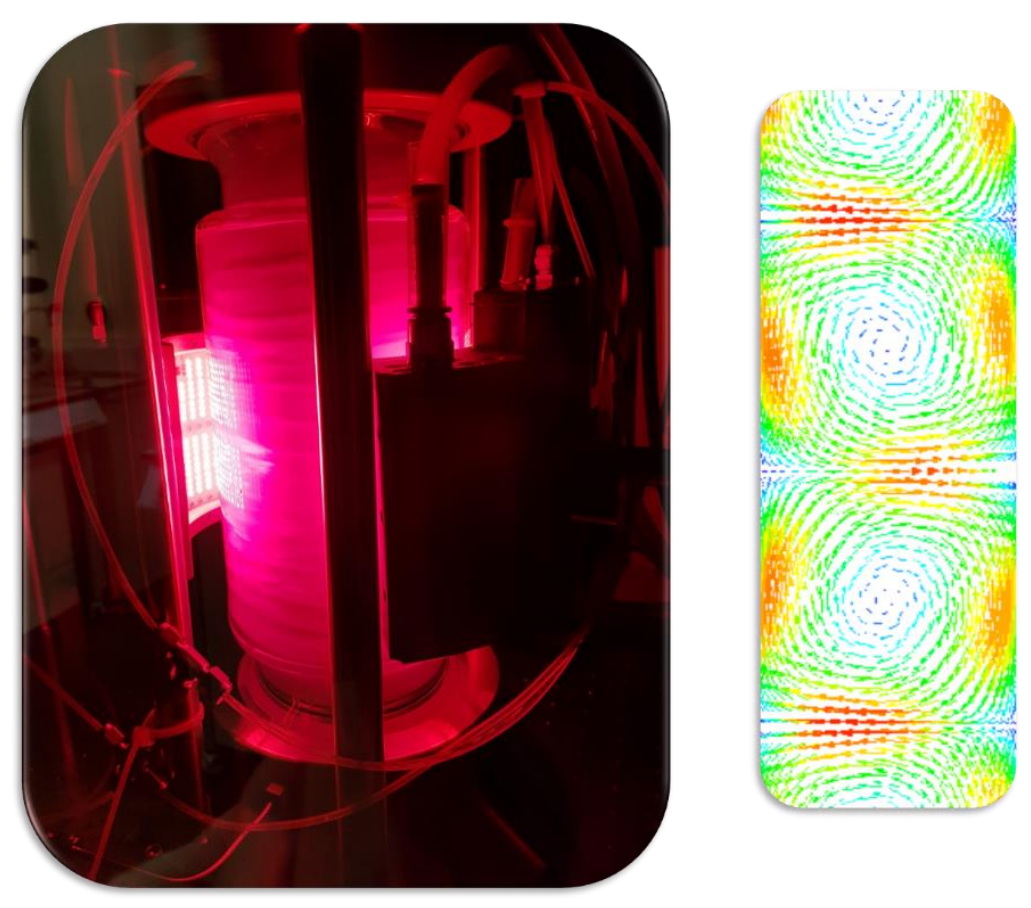




\section{Abstract}

We report the development of a scalable continuous Taylor vortex reactor for both UV and visible photochemistry. This builds on our recent report (Org. Process Res. Dev. 2017, 21, 1042) detailing a new approach to continuous visible photochemistry. Here we expand this by showing that our approach can also be applied to UV photochemistry and that either UV or visible photochemistry can be scaled-up using our design. We have achieved scale-up in productivity of over $300 \times$ with a visible light photo-oxidation that requires oxygen gas and $10 \times$ with a UV induced [2+2] cycloaddition obtaining scales of up to $7.45 \mathrm{~kg} \mathrm{day}^{-1}$ for the latter. Furthermore, we demonstrate that oxygen is efficiently taken up in to the reactions of singlet $\mathrm{O}_{2}$ and, for the examples examined, that near-stoichiometric quantities of oxygen can be used with little loss of reactor productivity. Furthermore, our design should scalable to substantially larger size as well as having the potential for scaling-out with reactors in parallel.

Keywords: Photochemistry, Photo-oxidation, Continuous Flow, Flow Chemistry, Singlet Oxygen, Vortex Reactor

\section{Introduction}

Sustainability is a key driver for both academic and industrial chemistry research and there is a need for continual interventions to drive this agenda if we are to have a major impact on delivering sustainable chemistry. ${ }^{1-4}$ The application of photochemistry to chemical processes is particularly appealing in this context as photons can be considered as 'green' reagents. Moreover, photochemistry can offer milder approaches for obtaining highly reactive intermediates and/or inducing strain into complexes which then allows access to molecules and scaffolds with structural complexity, that are often difficult to construct using other methodologies. ${ }^{5}$ Much of the current interest in applying photochemistry for synthesis has been focussed on visible-light-induced organic reactions, particularly in the area of photoredox catalysis, ${ }^{6-7}$ and somewhat less attention is being paid to UV photochemistry.

There are limitations to using photochemistry in an industrial setting, particularly in terms of light penetration, which make the scale-up of photochemical batch processes more difficult. However, the application of flow chemistry, continuous manufacturing and the associated 
technologies is becoming more widely adopted as alternative to traditional batch methods. ${ }^{8-}$ 10 Such approaches have attractions for photochemistry because they offer alternative scalable routes using chemistries that are often under-utilised in industrial settings. By using smaller footprint reactors only small volumes of the reaction mixture are processed at one time, meaning that the build-up of reactive or hazardous intermediates can be controlled more easily than in batch. Furthermore, several reactors can be linked in order to carry out a multiple steps in one streamlined process. ${ }^{11-13}$ Flow chemistry offers particular advantages for photochemistry, such as overcoming the limitations of light penetration, reactor fouling and over-irradiation which make the scale-up of batch processes more difficult and are detrimental to its wide-spread implementation. ${ }^{14-15}$

The development of flow photochemistry has been facilitated by devlopments in reactor design, for example, there are several reactor designs that address the effective and safe addition of gases such as the photochemically generated singlet oxygen. ${ }^{16-26}$ The recent development in applications of visible photochemistry has also, in part, been driven by advances in Light-Emitting Diodes (LEDs) ${ }^{27-28}$ which provide inexpensive high intensity visible light sources. LEDs emitting in the UVA region are also available but this technology is still nascent, with the LEDs having lower powers and higher cost, limiting so far the large-scale application of UV LEDs for photochemistry. Thus the development of larger-scale UV photochemical processes still depends on more conventional light sources such as Hg lamps. UV photochemistry involves the absorption of photons, in the wavelength region of $100-400$ $\mathrm{nm}$. In these reactions, the photons are often directly absorbed by the reagent molecule without the need for an additional catalyst. UV photochemistry is synthetically useful because it gives access to more unusual and strained motifs. Furthermore, these products can then be used in a range of subsequent transformations. ${ }^{29-32}$ An early innovation for continuous flow UV photochemistry consisted of a coil of Fluorinated Ethylene Propylene (FEP) wrapped around a transparent housing containing a Hg lamp. ${ }^{33}$ More recently, this tubular design have been transformed a high capacity Parallel Tube Flow Reactor (PTFR) for UV chemistry capable of producing multi-kilogram quantities of product in a single day. ${ }^{34}$ There are several other continuous photochemical reactor designs that have been recently disclosed in the literature. ${ }^{35}$ Our own contributions have involved the generation of thin films of reaction mixture for more efficient light penetration using either excimer lamps for discrete 
wavelength excitation, ${ }^{36}$ or high powered light sources with a modified rotary evaporator for visible and/or UV photochemistry. ${ }^{37-38}$

Of particular relevance to this paper is our recent report of a small-scale visible light reactor ${ }^{39}$ which exploited the use of Taylor vortices. ${ }^{40}$ In this design, the chemical reactor consists of a transparent cylindrical outer vessel with a second smooth cylinder fitted coaxially inside it. Between the two cylinders there is a relatively narrow gap which contains the reaction solution. The inner cylinder is rotated at relatively high speed (e.g. $4000 \mathrm{rpm}$ ), generating so called "Taylor" or "Taylor-Couette" vortices, toroidal vortices threaded around the inner rotating cylinder. The precise nature and intensity of the vortices is a function of both the dimensions of the reactor and the rotation speed. The first manifestation of our laboratoryscale continuous flow Taylor vortex reactor was intended primarily for reactions of singlet oxygen photo-generated by visible light. In addition, the reactor was capable of performing thermal oxidation reactions involving $\mathrm{O}_{2} \cdot{ }^{39}$ We combined several design features to construct a borosilicate reactor that was surrounded by high-power visible light LEDs and drew air in from the top of the vessel when it rotates as shown in Figure 1.
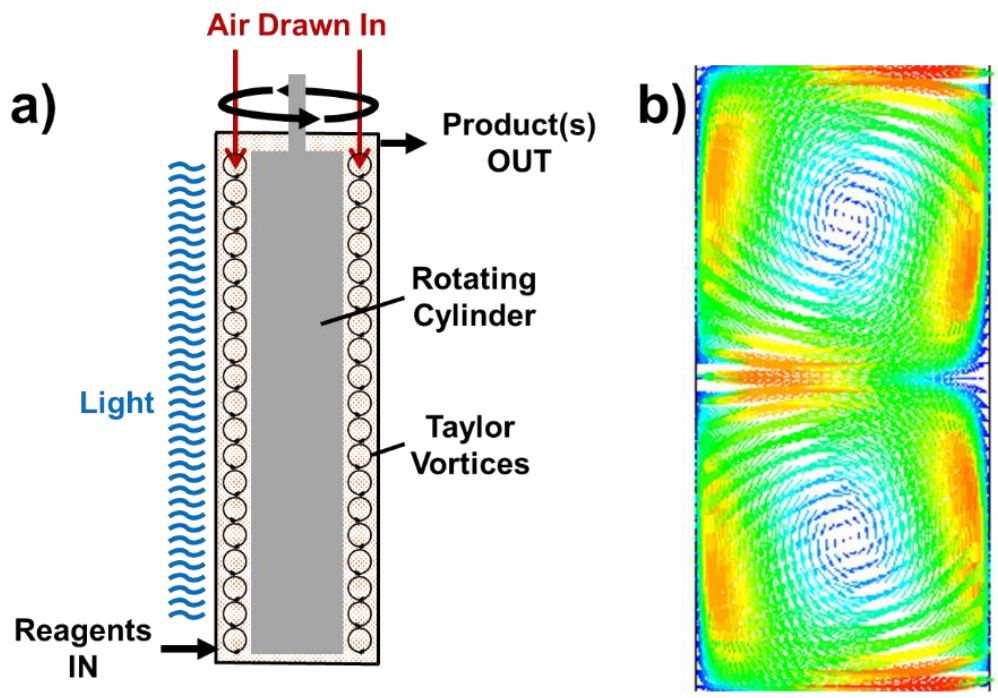

Figure 1. a) Simplified schematic depicting the cross section of the continuous flow visible light vortex reactor for reactions of singlet $\mathrm{O}_{2}$ showing the smooth rotor (gray) inside the glass vessel; b) Visualisation of the vortices generated in the reactor between the rotating cylinder and the static glass 
wall; modelled using computational fluid dynamics (CFD). The colors show a velocity gradient where regions shown in red are highest velocity and the regions in blue are lower velocity. ${ }^{39}$

In this report, we describe the scale-up of a continuous Taylor vortex reactor for photochemistry and demonstrate its potential as an adaptable, high throughput UV and visible light photochemical reactor for gram to kilogram scale reactions.

\section{Results and Discussion}

Following our initial report of a laboratory scale vortex reactor, we chose to demonstrate the scalability of this concept by developing a reactor that is approximately $20-30$ times the volume. As the generation of Taylor vortices in a fluid comes from a rotating cylinder inside a static cylinder, where the gap is small (i.e. a few $\mathrm{mm}$ ), one only needs to increase the size of the cylinders whilst maintaining the gap size to achieve a scaled up reactor. In theory, the height (or length, depending on orientation) of the reactor could be anything. Furthermore, as the radius of the rotating cylinder is increased the tangential velocity at the surface of the cylinder becomes greater. For example, in the simplest approximation, a cylinder of radius 10 $\mathrm{mm}$ rotating at $3000 \mathrm{rpm}$ has a tangential velocity of $3.1 \mathrm{~m} \mathrm{~s}^{-1}$ whereas a cylinder with radius of $50 \mathrm{~mm}$ rotating at the same $3000 \mathrm{rpm}$ has a tangential velocity of $15.5 \mathrm{~m} \mathrm{~s}^{-1}$. To keep the same tangential velocity as the $10 \mathrm{~mm}$ radius cylinder then the $50 \mathrm{~mm}$ radius cylinder need only to rotate at approx. $600 \mathrm{rpm}$. Inherently, this makes operation of a larger reactor less challenging as a larger (and possibly heavier) cylinder has to be rotated at only a fraction of the speed. However, one drawback of the slower rotation speed is that there is less air entrained from the atmosphere. In the smaller scale reactor we demonstrated that the amount of air drawn into the reactor increased with rotation speed (up to $4000 \mathrm{rpm}$ ). ${ }^{39}$ Although drawing air from the laboratory is obviously beneficial from one point of view because a pressurised gas cylinder is not needed for reactions involving oxygen, this approach does not offer control over the input of gases and limits the productivity of the reactor to the amount of oxygen entrained. With this in mind, for our scaled-up design, we opted to have the option of adding a gas flow into the reactor.

During the construction and design of the reactor, we used Computational Fluid Dynamics (CFD) modelling to identify the key parameters affecting the behaviour of the vortices 
between the two scales of reactor. Such vortices are usually characterised by the dimensionless 'Taylor number' but, in the case of our reactors, it quickly became clear that calculating the Taylor number in the standard way did not give a complete picture of the reactors. We concluded that the Taylor number, Ta, could not be used as a the sole measure of vortex strength. An important question to be decided by CFD was how fast the larger reactor should rotate. In this context, our CFD studies indicated that, when Ta is fixed at a value found for the small reactor (Ta $=$ ca. $7.03 \times 10^{6}$ calculated at $4000 \mathrm{rpm}$ for a $1 \mathrm{~mm}$ annulus), a rotation speed of $1300 \mathrm{rpm}$ would be required for the large reactor $(2 \mathrm{~mm}$ annulus) to generate vortices of a similar strength. Figure 2 shows the similarity between the vortices in the small reactor versus the large reactor where the strength of the vortices is maintained at the same level.
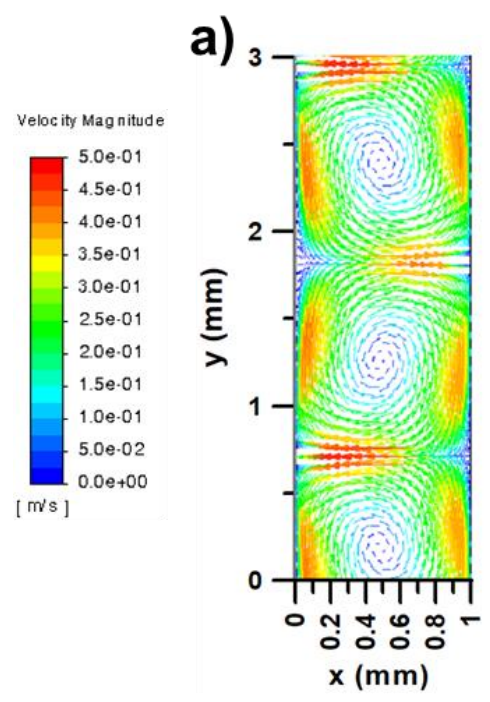

Small vortex (1 mm gap, $4000 \mathrm{rpm}$ )

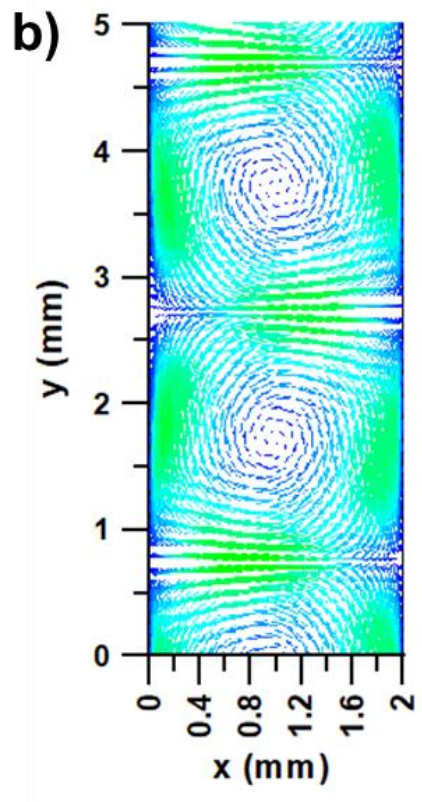

Large vortex (2 mm gap, $660 \mathrm{rpm}$ )

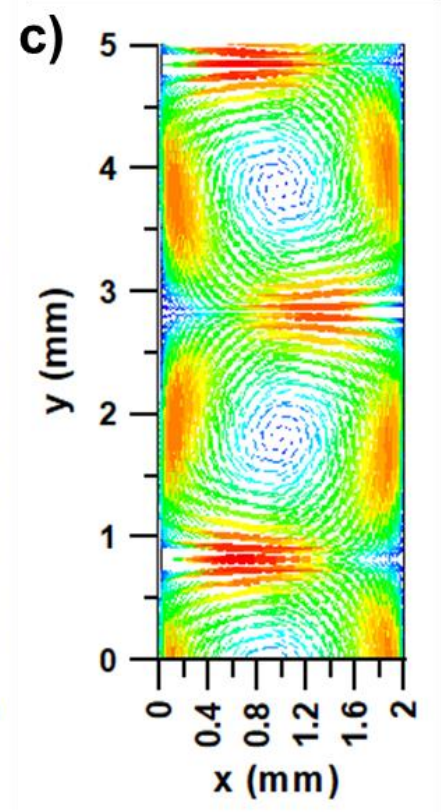

Large vortex (2 mm gap, $1300 \mathrm{rpm}$ )

Figure 2. Computational Fluid Dynamics (CFD) modelling comparing (a) the small vortex reactor ( $1 \mathrm{~mm}$ gap, $4000 \mathrm{rpm}$ ) and the large vortex reactor at two different speeds (b) (2 mm gap, $660 \mathrm{rpm}$ ) and (c) (2mm gap, $1300 \mathrm{rpm}$ ).

Initially, we considered designing the reactor as a $1 \mathrm{~L}$ round bottom jacketed reaction vessel with a rotor suspended from the top, similar in design to our original prototype reactor. Considering the weight of the rotor, we moved away from this design and decided to secure the rotor at each end to reduce as much as possible any issues (such as wobble) that could 
arise from the increased weight and size of the rotor. With this major constraint, we opted to use readily available standard glassware, namely an open-ended jacketed filter-tube, which can be obtained in a variety of sizes. The final design of the reactor (Figure 3 ) consists of the jacketed filter-tube which is sealed between a steel base and a polymer cap with a hollow polymer rotor inside. The rotation is driven from a motor that is mounted on top. Inlet ports for gas and liquids are bored through the steel base, while the outflow for gas/liquid is found in the polymer cap, where the liquid and gas can be drawn out of 4 ports equally spaced around the cap. Cooling is provided by a recirculating chiller that is connected to the jacketed glass vessel. In this reactor, the gap between the rotor and the glass vessel was made to be 2 $\mathrm{mm}$ and the reactor volume was $280 \mathrm{~mL}$. Further details of the reactor are given in the ESI.
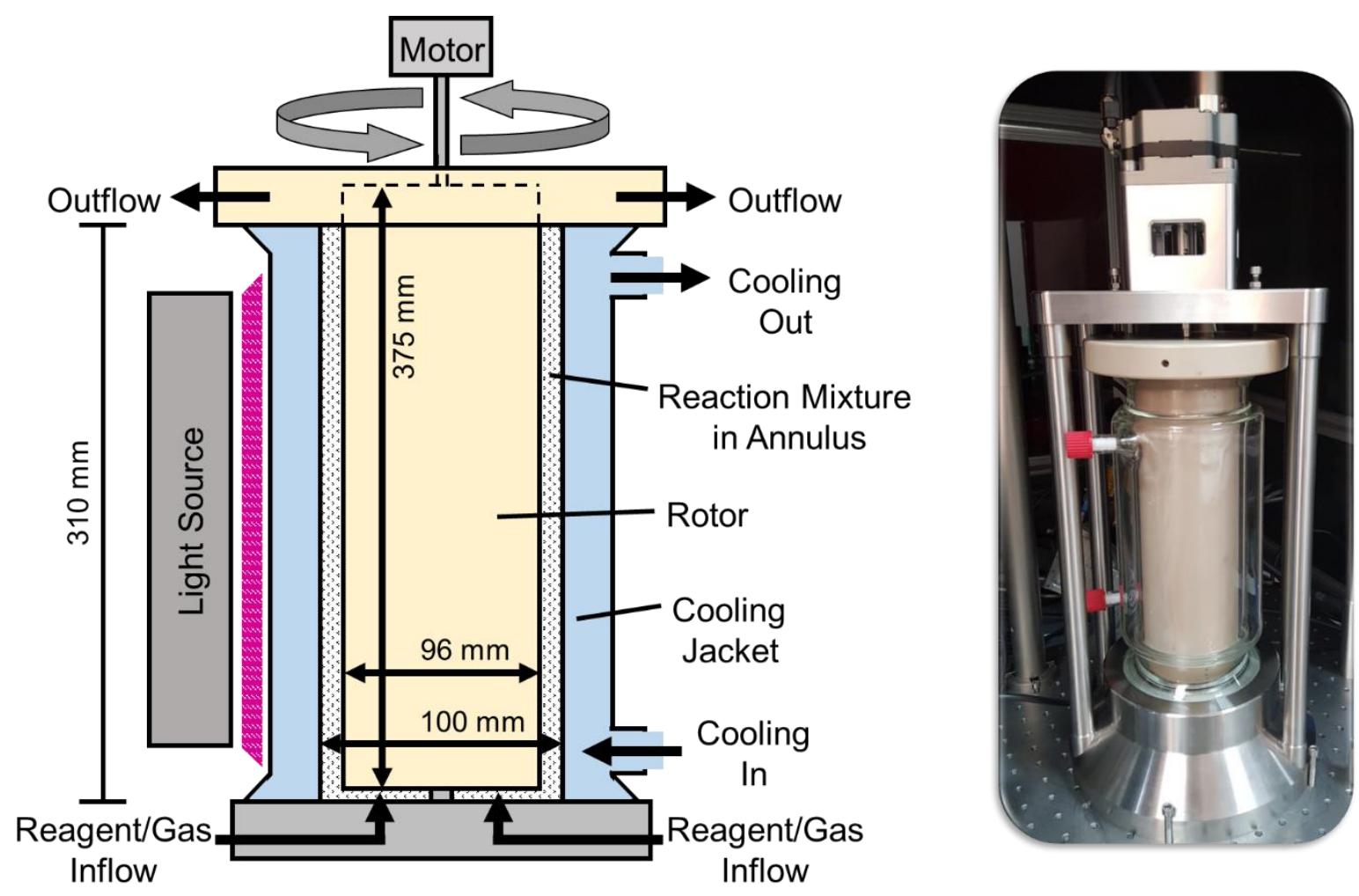

Figure 3. Left: Simplified cross section (not to scale) of the scaled-up vortex reactor showing key dimentions of the glassware and rotor, for details of construction, see ESI; Right: Photograph of the final reactor design. 
To demonstrate the versatility of the reactor we chose to study two reactions in the reactor, one requiring UV light and one requiring visible light and oxygen. In our previous paper we studied several visible light reactions involving singlet oxygen in the small reactor but had not studied UV reactions. For the visible light reaction, the photo-oxidation of citronellol $\mathbf{1}$ to its corresponding peroxides $\mathbf{2}$ and $\mathbf{3}$ was studied. ${ }^{41-43}$ Using the small reactor, in the set-up that was described previously, ${ }^{39}$ the reaction was run using ethanol as a solvent, rose bengal as the photosensitiser and the oxygen was taken from the air drawn in through the top of the reactor. The yield of $\mathbf{2} / 3$ was $73 \%$ at $0.5 \mathrm{~mL} \mathrm{~min}^{-1}$ and $95 \%$ at $0.25 \mathrm{~mL} \mathrm{~min}^{-1}$, which equates to a productivity of 4.1 and $6.4 \mathrm{~g} \mathrm{day}^{-1}$ respectively (Table 1 ).

As the large reactor is approximately 20 times larger in volume compared to the small reactor, the flow rate was scaled by 20 times from 0.5 to $10 \mathrm{~mL} \mathrm{~min}^{-1}$ and this was used as the starting point for tests in the large reactor. Increasing the amount of oxygen led to an increase in conversion/yield (Table 1, entry 3-5) and these initial results show that the reaction efficiency is dependent on the amount of oxygen delivered to the reactor (Table 1, entries 3-4) as increasing the air input rate led to an increase in yield. Note: in entries $3 \& 4$ the yield appears higher than the amount of oxygen supplied to the reactor; this was due to residual air dissolved in the starting solution. When oxygen is in excess the reaction goes to completion (Table 1, entries 5-6) but when 1 is in excess the amount of oxygen gives an approximation for the yield. For example, in the case of entry 7 (Table 1), $60 \mathrm{~mL} \mathrm{~min}^{-1} \mathrm{O}_{2}$ equates to approx. $2.45 \mathrm{mmol} \mathrm{min}^{-1}$ and as the substrate is provided at $4 \mathrm{mmol} \mathrm{min}-1$ ( $\left.20 \mathrm{~mL} \mathrm{~min}^{-1} \times 0.2 \mathrm{M}\right)$ therefore it would be expected that the yield of $2 / 3$ would be approx. $61 \%$, which experimentally was found to be $59 \%$ (see ESI for more details).

These results suggest that, at least in this reaction, the reactor is quite efficient in terms of the uptake of oxygen and large excesses of the gas can be avoided. Further reducing the stoichiometry of 1 to $\mathrm{O}_{2}$ (Table 1, entry 8), proved successful as with a minor excess of oxygen the efficiency of the reaction was still high. Further increasing the flow rate of the liquid and gas (Table 1, entry 9) boosted productivity, however the reaction did not reach completion. Increasing the photosensitiser concentration and the rotation speed (Table 1, entry $10 \& 11$ respectively) gave no significant change in the yield, suggesting that the reaction was at the limit of the light provided. Switching to higher power LEDs (Table 1, entry 12) enabled the reaction to reach completion and gave a yield of $92 \%$ which equates to the equivalent of 
nearly $2 \mathrm{~kg} \mathrm{day}^{-1}$. Provided that cooling is efficient enough to remove heat from the LEDs, the reactor can be operated for several hours without issue (with runs in this study lasting between $30 \mathrm{~min}-3 \mathrm{~h}$ ). We estimate that the residence time of the reactor for a flow rate of $40 \mathrm{~mL} \mathrm{~min}-1$ is approximately 7 minutes (based up on a volume of $280 \mathrm{~mL}$ ), however we are conducting further studies using CFD and experimental work to further investigate the residence time distribution. 
Table 1. Showing the results of the photo-oxidation of citronellol $\mathbf{1}$ in the small and large reactors.

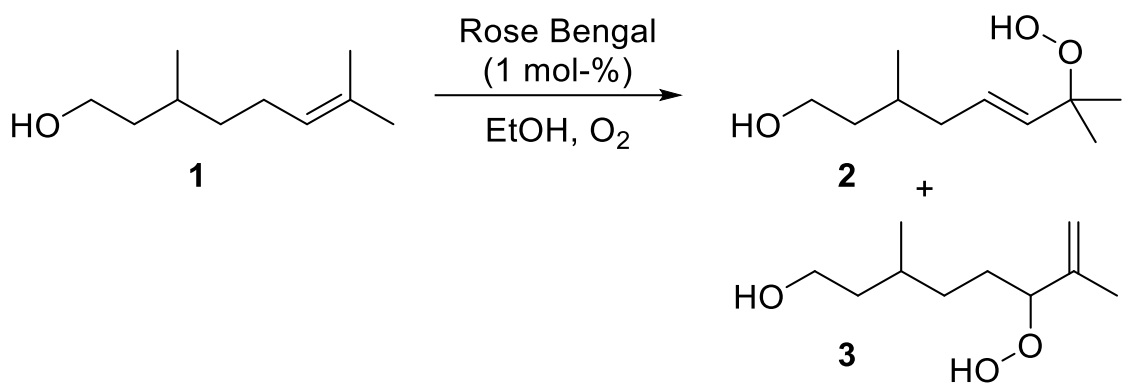

\begin{tabular}{|c|c|c|c|c|c|c|c|c|}
\hline Entry & $\begin{array}{l}\text { Flow Rate } \\
\left(\mathrm{mL} \min ^{-1}\right)\end{array}$ & $\begin{array}{c}\text { Conc. } \\
\text { (M) }\end{array}$ & $\begin{array}{l}\text { Gas Flow } \\
\left(\mathrm{mL} \mathrm{min}^{-1}\right)^{\mathrm{a}}\end{array}$ & $\begin{array}{c}\text { Ratio } \\
\text { of }\end{array}$ & $\begin{array}{l}\text { Rotation } \\
\text { (rpm) }\end{array}$ & $\begin{array}{l}\text { Yield } \\
2 / 3\end{array}$ & $\begin{array}{l}\text { Prod. (g } \\
\left.\text { day }^{-1}\right)^{d}\end{array}$ & $\begin{array}{l}\text { STY (g } \\
\text { day }^{-1}\end{array}$ \\
\hline & & & & $1: \mathrm{O}_{2}^{\mathrm{b}}$ & & $(\%)^{c}$ & & $\left.m L^{-1}\right)^{f}$ \\
\hline
\end{tabular}

\section{Small Reactor}

\begin{tabular}{ccccccccc}
\hline 1 & 0.5 & 0.1 & DI (air) & - & 4000 & 73 & 4.1 & 0.51 \\
2 & 0.25 & 0.1 & DI (air) & - & 4000 & 95 & 6.4 & 0.8 \\
\hline
\end{tabular}

Large Reactor

\begin{tabular}{lcccccccc}
\hline 3 & 10 & 0.1 & 10 (air) & $11: 1$ & 660 & 15 & 41 & 0.14 \\
4 & 10 & 0.1 & 60 (air) & $2: 1$ & 660 & 55 & 149 & 0.53 \\
5 & 10 & 0.1 & $60\left(\mathrm{O}_{2}\right)$ & $1.2: 5$ & 660 & 95 & 258 & 0.92 \\
6 & 20 & 0.1 & $60\left(\mathrm{O}_{2}\right)$ & $0.77: 1$ & 660 & 95 & 515 & 1.84 \\
7 & 20 & 0.2 & $60\left(\mathrm{O}_{2}\right)$ & $1.6: 1$ & 660 & 59 & 641 & 2.29 \\
8 & 20 & 0.2 & $100\left(\mathrm{O}_{2}\right)$ & $0.98: 1$ & 660 & 94 & 1020 & 3.64 \\
9 & 40 & 0.2 & $200\left(\mathrm{O}_{2}\right)$ & $0.98: 1$ & 660 & 78 & 1690 & 6.04 \\
$10^{\mathrm{e}}$ & 40 & 0.2 & $200\left(\mathrm{O}_{2}\right)$ & $0.98: 1$ & 660 & 74 & 1610 & 5.75 \\
11 & 40 & 0.2 & $200\left(\mathrm{O}_{2}\right)$ & $0.98: 1$ & 1320 & 76 & 1650 & 5.89 \\
12 & 40 & 0.2 & $200\left(\mathrm{O}_{2}\right)$ & $0.98: 1$ & 660 & 92 & 1995 & 7.13
\end{tabular}

$0.1 \mathrm{M}$ citronellol (1) in EtOH with $1 \mathrm{~mol}$ \% rose bengal; Reactor temp. $=25^{\circ} \mathrm{C}$. Small Reactor: $3 \times 65 \mathrm{~W}$ White LED blocks. Large Reactor: Entries 3-11 - $2 \times 300$ W LED arrays, entry $12-2 \times 1 \mathrm{~kW}$ LED arrays. a) DI = "Drawn in" and refers to the situation where air is drawn in to the reactor without any gases added to the reactor; Air flow rate in the large reactor was set using a mass flow controller. b) Ratio determined by calculating the $\mathrm{mmol} \mathrm{min}^{-1}$ of $\mathbf{1}$ and $\mathrm{mmol} \mathrm{min}^{-1}$ of $\mathrm{O}_{2}$ using the ideal gas equation. c) Yield of 2/3 was measured using ${ }^{1} \mathrm{H}$ NMR with biphenyl as an external standard. d) Projected productivity per day was calculated using [\{[(Conc. $\times$ Flow Rate)/1000] $\times 188.267\} \times 1440] \times Y i e l d$. e) with 2 mol-\% rose bengal. f) STY = Space time yield = Productivity / Reactor volume (based on the total volume of the reactor as opposed to the illuminated volume). 
Preliminary experimental validation of the modelling for single phase liquid reactions (i.e. without added gas) suggests that once the reactor is beyond the critical Taylor number $\left(\mathrm{Ta}_{\mathrm{c}}\right)$, which corresponds to a rotational speed of approximately $120 \mathrm{rpm}$ for this small diameter reactor (with a $1 \mathrm{~mm}$ annulus); For the large scale reactor with a $2 \mathrm{~mm}$ annular gap $\mathrm{Ta}_{\mathrm{c}}=$ approx. $20 \mathrm{rpm}$ ), any increase in mixing will not increase the yield any further, Figure 2. For reactions involving gas, the situation becomes more complex, as initially the gas occupies a volume in the reactor and the nature of the bubbles changes with rotation speed and the size of the annulus. In our small scale reactor the rotation speed must be well beyond that needed for $\mathrm{Ta}_{c}$ in order to efficiently draw in air; however it is likely that the Taylor number is not a significant factor in the drawing in of air. We are currently working on further understanding the behaviour of the reactor, in particular when gas is added and how this affects the fluid dynamics and the chemistry.

Changing to UV photochemistry, we began by studying the chemistry in the small reactor to evaluate the reactors performance. Intramolecular [2+2] photocycloadditions are becoming a benchmark for each reactors and tricyclic diones (so called Cookson's diones) such as 4 undergo a [2+2] photocycloaddition to yield diketones, such as $\mathbf{5}$, and have been investigated by both us and others. ${ }^{34,37,44}$ In both the small and the large reactor we employed high powered $\mathrm{Hg}$ lamps (1.4 kW or $2 \mathrm{~kW}$ units - see ESI for further details). In the small reactor, good results were obtained using a single $1.4 \mathrm{~kW}$ lamp, with complete conversion and $>99 \%$ yield at flow rates of 1-4 $\mathrm{mL} \mathrm{min}^{-1}$ (Table 2, entries 1-4). At $5 \mathrm{~mL} \mathrm{~min}^{-1}$, the conversion began

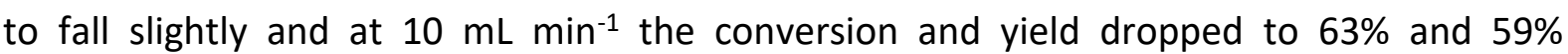
respectively (Table 2 , entries 5-6). Using this set-up and a borosilicate jacketed vessel gave a productivity of up to $30.9 \mathrm{~g} \mathrm{~h}^{-1}$ (742 $\left.\mathrm{g} \mathrm{day}^{-1}\right)$. 
Table 2. Conversion of $\mathbf{4}$ to $\mathbf{5}$ using the continuous flow vortex reactor under UV irradiation.

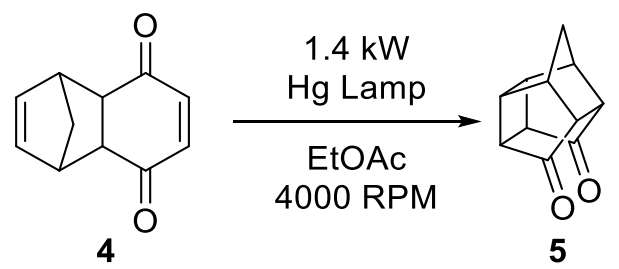

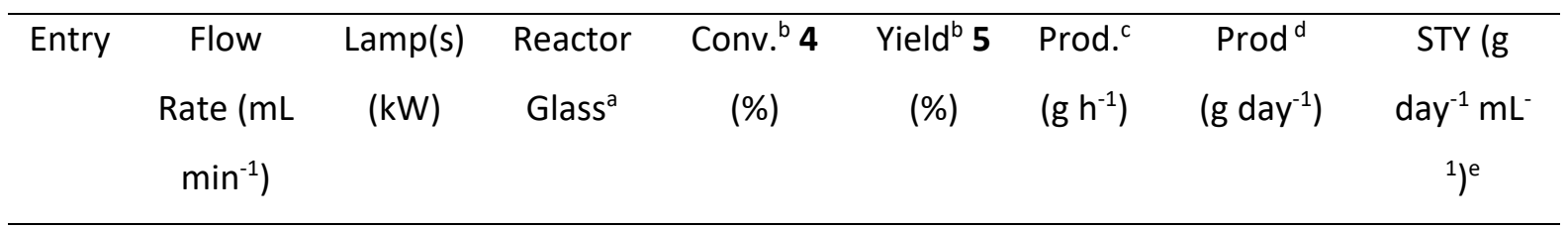

Small Reactor

\begin{tabular}{ccccccccc}
\hline 1 & 1.0 & $1(1.4)$ & B & 100 & $>99$ & 5.2 & 125 & 15.6 \\
2 & 2.0 & $1(1.4)$ & B & 100 & $>99$ & 10.5 & 252 & 31.5 \\
3 & 3.0 & $1(1.4)$ & B & 100 & $>99$ & 15.7 & 377 & 47.1 \\
4 & 4.0 & $1(1.4)$ & B & 100 & $>99$ & 20.9 & 502 & 62.8 \\
5 & 5.0 & $1(1.4)$ & B & 94 & 94 & 24.5 & 588 & 73.5 \\
6 & 10.0 & $1(1.4)$ & B & 63 & 59 & 30.9 & 742 & 92.8 \\
7 & 5.0 & $1(1.4)$ & Q & 99 & 94 & 24.5 & 588 & 53.5 \\
8 & 8.0 & $1(1.4)$ & Q & 90 & 89 & 37.2 & 893 & 81.2 \\
9 & 10.0 & $1(1.4)$ & Q & 74 & 74 & 38.7 & 929 & 84.5 \\
\hline & & & & Large Reactor & & & \\
\hline 10 & 40 & $1(1.4)$ & B & 52 & 50 & 104.5 & 2510 & 8.96 \\
11 & 40 & $2(2.8)$ & B & 85 & 85 & 177.7 & 4270 & 15.3 \\
12 & 60 & $2(4)$ & B & 99 & 99 & 310.4 & 7450 & 26.6 \\
\hline
\end{tabular}

Small Reactor: $40.5 \mathrm{M}$ in EtOAc, $5{ }^{\circ} \mathrm{C}, 4000 \mathrm{rpm}$, degassed with argon (bororsilicate vessel = $8 \mathrm{~mL}$, quartz vessel = $11 \mathrm{~mL}$ ). Large Reactor: $40.5 \mathrm{M}$ in EtOAc, $5^{\circ} \mathrm{C}, 660 \mathrm{rpm}$, degassed with argon, (borosilicate vessel $=280 \mathrm{~mL}$ ). a) $\mathrm{B}=$ Borosilicate (UV cutoff $=280$ $\mathrm{nm}$ ), $\mathrm{Q}=$ Quartz (UV cutoff = $190 \mathrm{~nm}$ ); For reactions in the quartz vessel, significant fouling was observed after 15-60 minutes of reaction time; b) Conversion and Yields were measured by ${ }^{1} \mathrm{H}$ NMR against biphenyl as an external standard; c) Projected Productivity is calculated by $\{[($ Conc. $\times$ Flow Rate $) / 1000] \times 174.199\} \times 60 \times$ Yield; d) Projected productivity $\times 24$. e) STY = Space time yield = Productivity / Reactor volume (based on the total volume of the reactor as opposed to the illuminated volume).

Switching the jacketed glass vessel from borosilicate to one made of quartz opened up the possibility of capturing more light. However it exposes the chemistry to higher energy UV light which can cause fouling of the reactor. At $5 \mathrm{~mL} \mathrm{~min}{ }^{-1}$ there was little difference between the two sets of glassware (Table 2, entries 5 \& 7), but when increasing the flow rate to 8 and 10 $\mathrm{mL} \mathrm{min}^{-1}$ the increase in yield with quartz was more apparent (Table 2, entries 8-9) compared 
to borosilicate (Table 2, entry 6). With the reactions in quartz, however there was a build up of fouling on the quartz that gradually reduced both the conversion and yield when a reaction was run continuously over the period of an hour. In this case, the fouling that built up around the reactor, was a yellow film which could be removed by cleaning (see ESI for further details). No fouling of the reactor was apparent for this reaction when the borosilicate reactor was used. Moving to the large reactor, the capture of light was expected to be more efficient compared to the small reactor, as the geometry of the reactor is more suited to the size of the $\mathrm{Hg}$ lamps used in the study. Initially a single $1.4 \mathrm{~kW}$ lamp was used with a flow rate of 40 $\mathrm{mL} \mathrm{min}^{-1}$ (Table 2, entry 10), which yielded $50 \%$ of $\mathbf{5}$, and when a second $1.4 \mathrm{~kW}$ lamp was added ( $2.8 \mathrm{~kW}$ in total) the yield increased to $85 \%$ (Table 2, entry 11$)$, with a projected productivity of over $4.2 \mathrm{~kg}$ per day. When $4 \mathrm{~kW}(2 \times 2 \mathrm{~kW})$ of lamp power was used with 60 $\mathrm{mL} \mathrm{min}^{-1}$ quantitative conversion of 4 to 5 was observed with a projected productivity of nearly $7.5 \mathrm{~kg} \mathrm{day}^{-1}$.

\section{Conclusions}

A scalable continuous Taylor vortex reactor for photochemistry has been developed, from our initial prototype visible light reactor. The scaled up reactor has been validated with CFD modelling. Scale-up in productivity of over $300 \times$ has been achieved with visible light photooxidation chemistry that requires oxygen gas with scales of upto nearly $2 \mathrm{~kg} \mathrm{day}^{-1}$ in the large reactor compared to $6.4 \mathrm{~g} \mathrm{day}^{-1}$ in the small reactor (using air drawn in). Furthermore, the efficiency of oxygen uptake by the reactor has been exemplified by the use of near stoichiometric oxygen with very little loss of efficiency in the reaction. Additionally the reactor has been run for several hours with no issue. UV photochemistry was studied in our reactor for the first time and performed well in both the small (up to $742 \mathrm{~g} \mathrm{day}^{-1}$ in borosilicate) and the large reactor (up to $7.45 \mathrm{~kg} \mathrm{day}^{-1}$ ) with scale up in production by a factor of 10 between the small and large reactors (in borosilicate). Nevertheless, these values could probably be increased further. 


\section{Acknowledgements}

We thank EPSRC grant (EP/P013341/1) and Industrialization of Science and Technology Project, China (2014A35001-2) for financial support. We thank Prof. Kevin Booker-Milburn, Dr Charlotte Clark, Dr Calum Welsh, Dr Marcos Veguillas Hernando and Dr Rowena Howie for their useful input. The authors also wish to thank Richard Meehan, Richard Wilson, Ben Clarke, Matthew McAdam, David Lichfield, Martin Dellar and Mark Guyler for their technical support.

\section{Supporting Information}

Further information, including details of the reactor, experimental procedures and representative ${ }^{1} \mathrm{H}$ NMR spectra are provided in the Electronic Supporting Information (ESI).

\section{References}

1. Poliakoff, M.; Licence, P.; George, M. W., A New Approach to Sustainability: A Moore's Law for Chemistry. Angew. Chem. Int. Ed. 2018, 57 (39), 12590-12591.

2. Poliakoff, M.; Licence, P.; George, M. W., UN sustainable development goals: How can sustainable/green chemistry contribute? By doing things differently Curr. Opin. Green Sustain. Chem. 2018, 13, 146-149.

3. Poliakoff, M.; George, M. W., Manufacturing chemicals with light: any role in the circular economy? Phil. Trans. R. Soc. A, 2019, in press, doi:10.1098/not yet assigned.

4. Keijer, T.; Bakker, V.; Slootweg, J. C., Circular chemistry to enable a circular economy. Nat. Chem., 2019. 11, 190-195.

5. Kärkäs, M. D.; Porco, J. A.; Stephenson, C. R. J., Photochemical Approaches to Complex Chemotypes: Applications in Natural Product Synthesis. Chem. Rev. 2016, 116 (17), 9683-9747.

6. Skubi, K. L.; Blum, T. R.; Yoon, T. P., Dual Catalysis Strategies in Photochemical Synthesis. Chem. Rev. 2016, 116 (17), 10035-10074.

7. Romero, N. A.; Nicewicz, D. A., Organic Photoredox Catalysis. Chem. Rev. 2016, 116 (17), 1007510166.

8. Plutschack, M. B.; Pieber, B.; Gilmore, K.; Seeberger, P. H., The Hitchhiker's Guide to Flow Chemistry. Chem. Rev. 2017, 117 (18), 11796-11893.

9. Cambie, D.; Bottecchia, C.; Straathof, N. J. W.; Hessel, V.; Noel, T., Applications of ContinuousFlow Photochemistry in Organic Synthesis, Material Science, and Water Treatment. Chem. Rev. 2016, 116 (17), 10276-10341.

10. Elliott, L. D.; Knowles, J. P.; Koovits, P. J.; Maskill, K. G.; Ralph, M. J.; Lejeune, G.; Edwards, L. J.; Robinson, R. I.; Clemens, I. R.; Cox, B.; Pascoe, D. D.; Koch, G.; Eberle, M.; Berry, M. B.; BookerMilburn, K. I., Batch versus flow photochemistry: a revealing comparison of yield and productivity. Chem-Eur. J. 2014, 20 (46), 15226-15232.

11. Britton, J.; Raston, C. L., Multi-step continuous-flow synthesis. Chem. Soc. Rev. 2017, 46 (5), 12501271. 
12. Laroche, B.; Saito, Y.; Ishitani, H.; Kobayashi, S., Basic Anion-Exchange Resin-Catalyzed Aldol Condensation of Aromatic Ketones with Aldehydes in Continuous Flow. Org. Process Res. Dev. 2019, 23 (5), 961-967.

13. Porta, R.; Benaglia, M.; Puglisi, A., Flow Chemistry: Recent Developments in the Synthesis of Pharmaceutical Products. Org. Process Res. Dev. 2016, 20 (1), 2-25.

14. Politano, F.; Oksdath-Mansilla, G., Light on the Horizon: Current Research and Future Perspectives in Flow Photochemistry. Org. Process Res. Dev. 2018, 22 (9), 1045-1062.

15. André, J.; Viriot, M.; Villermaux J. New developments in photochemical technology. Pure \& Appl. Chem. 1986, 58 (6), 907-916.

16. Wu, L. Q.; Lee, D. S.; Boufroura, H.; Poliakoff, M.; George, M. W., Photooxidation of Fulvenes in a Continuous Flow Photoreactor using Carbon Dioxide as a Solvent. ChemPhotoChem 2018, 2 (7), 580-585.

17. Wu, L. Q.; Abada, Z.; Lee, D. S.; Poliakoff, M.; George, M. W., Combining engineering and chemistry for the selective continuous production of four different oxygenated compounds by photo-oxidation of cyclopentadiene using liquid and supercritical $\mathrm{CO} 2$ as solvents. Tetrahedron 2018, 74 (25), 3107-3112.

18. Ioannou, G. I.; Montagnon, T.; Kalaitzakis, D.; Pergantis, S. A.; Vassilikogiannakis, G., One-Pot Synthesis of Diverse $\gamma$-Lactam Scaffolds Facilitated by a Nebulizer-Based Continuous Flow Photoreactor. ChemPhotoChem 2017, 1 (5), 173-177.

19. Amara, Z.; Bellamy, J. F. B.; Horvath, R.; Miller, S. J.; Beeby, A.; Burgard, A.; Rossen, K.; Poliakoff, M.; George, M. W., Applying green chemistry to the photochemical route to artemisinin. Nat. Chem. 2015, 7 (6), 489-495.

20. Bourne, R. A.; Han, X.; Chapman, A. O.; Arrowsmith, N. J.; Kawanami, H.; Poliakoff, M.; George, M. W.; Homogeneous photochemical oxidation via singlet $\mathrm{O}_{2}$ in supercritical $\mathrm{CO}_{2}$. Chem. Commun., 2008, 4457-4459.

21. Van Gerven, T.; Mul, G.; Moulijn, J.; Stankiewicz, A., A review of intensification of photocatalytic processes. Chem. Eng. Process. 2007, 46 (9), 781-789.

22. Jähnisch, K.; Dingerdissen, U., Photochemical Generation and [4+2]-Cycloaddition of Singlet Oxygen in a Falling-Film Micro Reactor. Chem. Eng. Tech. 2005, 28 (4), 426-427.

23. Dionysiou, D. D.; Balasubramanian, G.; Suidan, M. T.; Khodadoust, A. P.; Baudin, I.; Laîné, J. M., Rotating Disk Photocatalytic Reactor: Development, Characterization, ad Evaluation for the Destruction of Organic Pollutants in Water. Water Res. 2000, 34 (11), 2927-2940.

24. Barberis, K.; Howarth, C. R., Reactivity Studies In The Ozonolysis Of Pollutants Using A Perforated Spinning Disc Reactor To Enhance Mass Transfer. Ozone: Sci. Eng. 1991, 13 (5), 501-519.

25. Gemoets, H. P. L.; Su, Y.; Shang, M.; Hessel, V.; Luque, R.; Noël, T., Liquid phase oxidation chemistry in continuous-flow microreactors. Chem. Soc. Rev. 2016, 45, 83-117.

26. Hone, C. A.; Roberge, D. M.; Kappe, C. O., The Use of Molecular Oxygen in Pharmaceutical Manufacturing: Is Flow the Way to Go? ChemSusChem 2017, 10 (1), 32-41.

27. Sender, M.; Ziegenbalg, D., Light Sources for Photochemical Processes - Estimation of Technological Potentials. Chem. Ing. Tech. 2017, 89 (9), 1159-1173.

28. Bonfield, H. E.; Mercer, K.; Diaz-Rodriguez, A.; Cook, G. C.; McKay, B. S. J.; Slade, P.; Taylor, G. M.; Ooi, W. X.; Williams, J. D.; Roberts, J. P. M.; Murphy, J. A.; Schmermund, L.; Kroutil, W.; Mielke, T.; Cartwright, J.; Grogan, G.; Edwards, L. J., The Right Light: De Novo Design of a Robust Modular Photochemical Reactor for Optimum Batch and Flow Chemistry. ChemPhotoChem 2019, DOI:10.1002/cptc.201900203.

29. Remy, R.; Bochet, C. G., Arene-Alkene Cycloaddition. Chem. Rev. 2016, 116 (17), 9816-9849.

30. Ravelli, D.; Protti, S.; Fagnoni, M., Carbon-Carbon Bond Forming Reactions via Photogenerated Intermediates. Chem. Rev. 2016, 116 (17), 9850-9913.

31. Poplata, S.; Troster, A.; Zou, Y.; Bach, T., Recent Advances in the Synthesis of Cyclobutanes by Olefin [2 + 2] Photocycloaddition Reactions. Chem. Rev. 2016, 116 (17), 9748-9815. 
32. Hoffmann, N., Photochemical Reactions as Key Steps in Organic Synthesis. Chem. Rev. 2008, 108 (3), 1052-1103.

33. Hook, B. D. A.; Dohle, W.; Hirst, P. R.; Pickworth, M.; Berry, M. B.; Booker-Milburn, K. I., A practical flow reactor for continuous organic photochemistry. J. Org. Chem. 2005, 70 (19), 7558-7564.

34. Elliott, L. D.; Berry, M.; Harji, B.; Klauber, D.; Leonard, J.; Booker-Milburn, K. I., A Small-Footprint, High-Capacity Flow Reactor for UV Photochemical Synthesis on the Kilogram Scale. Org. Process Res. Dev. 2016, 20 (10), 1806-1811.

35. Telmesani, R.; Park, S.; Lynch-Colameta, T.; Beeler, A., [2+2] Photocycloaddition of Cinnamates in Flow and Development of a Thiourea Catalyst. Angew. Chem. Int. Ed. 2015, 54 (39), 11521-11525.

36. DeLaney, E. N.; Lee, D. S.; Elliott, L. D.; Jin, J.; Booker-Milburn, K. I.; Poliakoff, M.; George, M. W., A laboratory-scale annular continuous flow reactor for UV photochemistry using excimer lamps for discrete wavelength excitation and its use in a wavelength study of a photodecarboxlyative cyclisation. Green Chem. 2017, 19 (6), 1431-1438.

37. Clark, C. A.; Lee, D. S.; Pickering, S. J.; Poliakoff, M.; George, M. W., UV PhotoVap: Demonstrating How a Simple and Versatile Reactor Based on a Conventional Rotary Evaporator Can Be Used for UV Photochemistry. Org. Process Res. Dev. 2018, 22 (5), 595-599.

38. Clark, C. A.; Lee, D. S.; Pickering, S. J.; Poliakoff, M.; George, M. W., A Simple and Versatile Reactor for Photochemistry. Org. Process Res. Dev. 2016, 20, 1792-1798.

39. Lee, D. S.; Amara, Z.; Clark, C. A.; Xu, Z. Y.; Kakimpa, B.; Morvan, H. P.; Pickering, S. J.; Poliakoff, M.; George, M. W., Continuous Photo-Oxidation in a Vortex Reactor: Efficient Operations Using Air Drawn from the Laboratory. Org. Process Res. Dev. 2017, 21 (7), 1042-1050.

40. White, F. M., Fluid Mechanics. McGraw Hill, 2011: 2011; Vol. 7.

41. Hamami, Z. E.; Vanoye, L.; Fongarland, P.; de Bellefon, C.; Favre-Réguillon, A., Improved Reactor Productivity for the Safe Photo-Oxidation of Citronellol Under Visible Light LED Irradiation. ChemPhotoChem, 2019, 3 (3), 122-128.

42. Lumley, E. K.; Dyer, C. E.; Pamme, N.; Boyle, R. W., Comparison of Photo-oxidation Reactions in Batch and a New Photosensitizer-Immobilized Microfluidic Device. Org. Lett. 2012, 14 (22), 5724 5727.

43. Maurya, R. A.; Park, C. P.; Kim, D., Triple-channel microreactor for biphasic gas-liquid reactions: Photosensitized oxygenations. Beilstein J. Org. Chem. 2011, 7, 1158-1163.

44. Elliott, L. D.; Knowles, J. P.; Stacey, C. S.; Klauber, D. J.; Booker-Milburn, K. I., Using batch reactor results to calculate optimal flow rates for the scale-up of UV photochemical reactions. React. Chem. Eng. 2018, 3, 86-93. 